\title{
Collaborative Spectrum Sensing under Suburban Environments
}

\author{
Aamir Zeb Shaikh \\ Department of Telecommunications Engineering, \\ NED University of Engineering \& Technology, \\ Karachi 75270. Pakistan
}

\author{
Dr. Talat Altaf \\ Department of Electrical Engineering, \\ NED University of Engineering \& Technology, \\ Karachi 75270. Pakistan
}

\begin{abstract}
Collaborative spectrum sensing for detection of white spaces helps in realizing reliable and efficient spectrum sensing algorithms, which results in efficient usage of primary spectrum in secondary fashion. Collaboration among cognitive radios improves probability of detecting a spectral hole as well as sensing time. Available literature, in this domain, uses Gudmundson's exponential correlation model for correlated lognormal shadowing under both urban and suburban environments. However, empirical measurements verify that the suburban environment can better be modeled through double exponential correlation model under suburban environments in comparison to Gudmundson's exponential correlation model. Collaboration among independent sensors provides diversity gains. Asymptotic detection probability for collaborating users under suburban environments using double exponential correlation model has been derived. Also, the Region of Convergence performance of collaborative detection is presented which agrees well with analytical derivations.
\end{abstract}

Keywords - collaborative spectrum sensing; suburban environment; asymptotic analysis; cognitive radio; opportunistic access.

\section{INTRODUCTION}

Cognitive radio is a revolutionary concept that aims to utilize licensed RF spectrum in an unlicensed/opportunistic fashion [1]. The successful opportunistic usage of spectrum requires information about unused spectrum bands termed as white spaces. This can be accomplished through Beaconassisted techniques and spectrum sensing algorithms. In Beacon-assisted based methods [2], primary user / licensed user transmits a beacon signal to cognitive users (opportunistic users) regarding available white-space on particular time and frequency bands that is decoded by the secondary users for successful exploitation of those spectral holes (white spaces). In spectrum sensing based techniques [3]; cognitive radio users detect white spaces (either individually or collaboratively) and exploit the identified bands in opportunistic fashion.

Spectrum sensing methods can be distributed in three categories i.e. transmitter, receiver and interferencetemperature based detection algorithms [3]. From these, transmitter detection based methods are a preferred way of sensing for presence/absence of spectral holes. These methods can be implemented through various techniques including coherent detection, feature detection and energy based detection algorithms [4]. Coherent sensor is an optimal linear detector for known primary signals in presence of white Gaussian noise [5]. However, detector implementation requires demodulation of received signals for achieving the optimal gains. Cognitive Radio operates in an opportunistic fashion that can operate in licensed as well as unlicensed bands and thus, it is quite difficult to demodulate each received signal before deciding in favor of presence/absence of a primary user. So, coherent sensor is generally not a preferred method of spectrum sensing. Cyclostationary feature based detector is an efficient and reliable method of spectrum sensing. These detectors compute Spectral Correlation function of received signals which serves as the signature of the particular signals. Thus, these detectors can easily distinguish between primary user signals, noise and other interfering sources (by using the features of corresponding signatures). However, these gains are achieved on the basis of exact licensed user information as well as received computational complexity.

On the other hand, energy sensing is a semi-blind method of detection of primary users [6]. Energy spectrum sensor computes the energy of the received signal samples and compares with a pre-set threshold. Setting of threshold requires noise information only. Computational simplicity makes these detectors a preferred choice for spectrum sensing cognitive radios. We use semi-blind algorithm i.e. energy detector for sensing the white spaces in our cognitive radio network under suburban environment.

Collaboration among secondary radios provides diversity gains. In [7] it is shown that under independent and identically distributed (i.i.d.) shadowing the detection probability can be significantly improved by exploiting user-collaboration. However, due to correlated shadowing the diversity gains are reduced [8].

In previous works, the detection probability (or misseddetection) for cognitive radio applications is computed using Gudmundson's exponential correlation model [9] under both urban and suburban environments. However, the results demonstrated by using real-time received signal measurement campaign performed by authors [10], analyze that the exponential correlation model best fits the autocorrelation function under urban environments but the results under suburban environments better follow double exponential model.

This paper considers the application of double exponential correlation model under suburban environments for cognitive radio applications. Asymptotic probability of detection is also derived. The simulation results verify the improvement in 
detection probability under double exponential correlation model in comparison to Gudmundson's correlation model under suburban environment.

The paper is organized as follows. The proposed system model and a brief discussion on the double correlation model are presented in Section II. In addition to that, this section also includes the derivation of asymptotic probability of detection using double exponential model under suburban environments. Section III presents the evaluation of detection probability under given environment conditions. Section IV concludes the paper in addition to a brief discussion on future work.

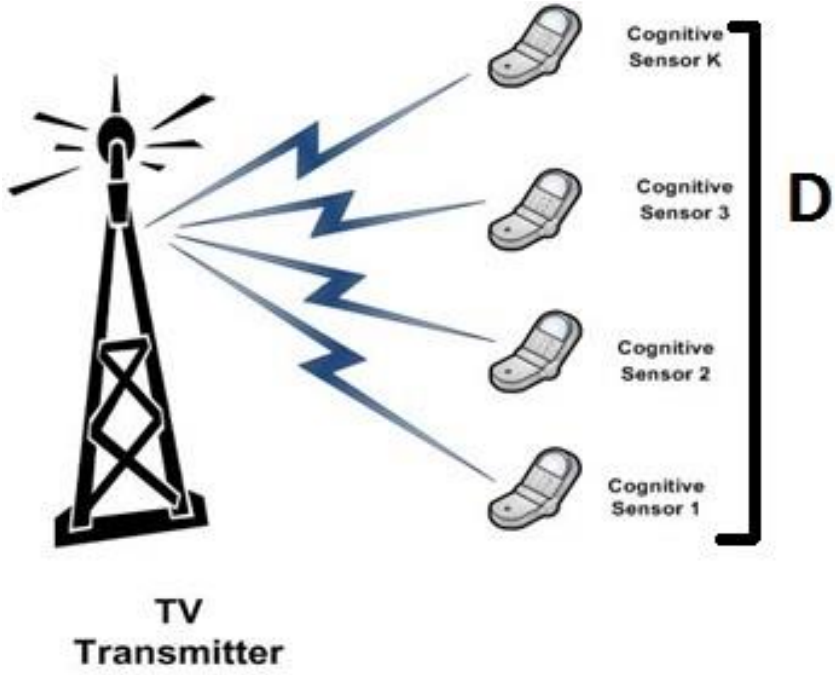

Fig. 1. Cognitive radio network, sensing TV Transmitter

\section{SYSTEM MODEL}

The proposed spectrum sensing scenario in this paper includes a TV transmitter as primary/licensed radio network and the secondary radio network consists of a large number of cognitive sensors, detecting primary transmissions collaboratively, as shown in Figure [1]. The received signal energy from primary transmitter to cognitive sensor can be represented with $\mathrm{y}(\mathrm{n})$ that can be defined as a binary hypothesis testing rule:

$$
y(n)=\left\{\begin{array}{cc}
\mathbf{n}_{\mathbf{i}}(\mathbf{n}) & ; H_{0} \\
\mathbf{h}_{\mathbf{i}}(\mathbf{n}) \mathbf{x}_{\mathbf{i}}(\mathbf{n})+\mathbf{n}_{\mathbf{i}}(\mathbf{n}) & ; H_{1}
\end{array}\right.
$$

$\mathrm{n}=0,1,2,3, \ldots, \mathrm{k}$ shows sample number of received signal, $\quad \mathrm{n}_{\mathrm{i}}(\mathrm{n})$ represents samples of AWGN, $n_{i}(n) \sim \square\left(0, \sigma_{n}{ }^{2}\right) \quad$ and $\quad \mathrm{h}_{\mathrm{i}}(\mathrm{n})$ shows fading channel coefficients, $h_{i}(n) \sim \square\left(0, \sigma_{h}^{2}\right)$ and $\mathrm{x}_{\mathrm{i}}(\mathrm{n})$ represents the Primary User signal samples. $\mathrm{H}_{0}$ hypothesis represents presence of a spectral hole while $\mathrm{H}_{1}$ represents the presence of a primary user signal.

Probability of detection $\left(\mathrm{P}_{\mathrm{D}}\right)$ represents the presence of a legitimate user (a.k.a. primary user), probability of misseddetection $\left(\mathrm{P}_{\mathrm{MD}}\right)$ shows that the detector flags as the presence of a legitimate user while in actually it is absent and the false alarm probability $\left(\mathrm{P}_{\mathrm{FA}}\right)$ represents the absence of a legitimate user, however, due to erroneous noise estimation or other errors the detector flags the presence of a primary user.

Thus, received signal vector y can be defined as a multivariate Gaussian random variable with following distribution:

$$
\mathbf{y} \sim \begin{cases}\square\left(\boldsymbol{\mu}_{0}, \sigma^{2} \Sigma\right) & ; H_{0} \\ \square\left(\boldsymbol{\mu}_{1}, \sigma^{2} \Sigma\right) & ; H_{1}\end{cases}
$$

Where $\mu_{0}$ and $\mu_{1} \in \square^{K \times 1}$ represent mean of received signal while $\sum \in \square^{K \times K}$ is representing the common covariance matrix of y under both null and alternative hypotheses i.e. $H_{0}$ and $H_{1}$. Mean and Covariance Matrices can be defined as:

$$
\boldsymbol{\mu}_{0}=\sigma_{n}^{2} \times \mathbf{1}, \quad \boldsymbol{\mu}_{1}=\left(P_{s}+\sigma_{n}^{2}\right) \times \mathbf{1}
$$

$\sigma_{n}^{2}$ is the mean signal power of received signal, $\mathrm{P}_{\mathrm{s}}$ is the mean signal power of the primary transmitter, received at cognitive sensor , ' 1 ' shows the vector of $1 \mathrm{~s}, \mu_{0}$ is the mean, whereas common covariance matrix is defined as:

$$
\Sigma=\frac{P_{S}}{m} \boldsymbol{\Lambda}+\frac{\sigma_{n}^{4}}{m} \mathbf{I}_{\mathbf{K}}
$$

$m=\tau \varpi$, Where $\tau$ represents the sensing time and $\varpi$ shows the bandwidth. $\Lambda$ represents double exponential correlation covariance matrix with $\mathrm{k} x \mathrm{k}$ measurements.

$$
\Lambda_{a, b}=\xi^{|a-b|}, a, b=1,2,3,4, \ldots \ldots . k
$$

\section{A. Channel Model}

We use double exponential model for suburban environments [10], which is given by following equation:

$$
\xi=h e^{-d / d_{A 1}}+(1-h) e^{-d / d_{A 2}}
$$

Where $\mathrm{d}_{\mathrm{A} 1}, \mathrm{~d}_{\mathrm{A} 2}$ are the short and long correlation distances, $h$ shows the strength between short and long correlation distance and d represents the distance travelled by the cognitive user.

Considering the case of secondary users with onedimensional distribution within a fixed distance $\mathrm{D}$ as in (i.e. $d=D /(k-1))$ where k represents the number of sensing.

\section{B. Hypothesis Testing}

It is assumed that the received signal power is less than the noise power as described in IEEE 802.22 [12]. The probability density function (PDF) of $\mathbf{y}$ under both null and alternate hypothesis is given by:

$$
\begin{aligned}
& f_{0}(\mathbf{y})=\frac{1}{(2 \pi)^{\frac{k}{2}}\left|\Sigma_{0}\right|^{\frac{1}{2}}} \exp \left\{-\frac{1}{2}\left(\mathbf{y}-\boldsymbol{\mu}_{0}\right)^{T} \Sigma_{0}^{-1}\left(\mathbf{y}-\boldsymbol{\mu}_{0}\right)\right\} \\
& f_{1}(\mathbf{y})=\frac{1}{(2 \pi)^{\frac{k}{2}}\left|\Sigma_{1}\right|^{\frac{1}{2}}} \exp \left\{-\frac{1}{2}\left(\mathbf{y}-\boldsymbol{\mu}_{1}\right)^{T} \Sigma_{1}^{-1}\left(\mathbf{y}-\boldsymbol{\mu}_{1}\right)\right\}
\end{aligned}
$$

Thus, Likelihood Ratio test can be evaluated as: 


$$
\begin{aligned}
& \Upsilon(\mathbf{y})=\frac{\left(\mathbf{x} ; H_{1}\right)}{\left(\mathbf{x} ; H_{0}\right)} \\
& \frac{1}{\underline{k} 1} \exp \left\{-\frac{1}{2}\left(\mathbf{y}-\mu_{1}\right)^{T} \Sigma_{1}^{-1}\left(\mathbf{y}-\mu_{1}\right)\right\} \\
& =\frac{(2 \pi)^{\frac{k}{2}}\left|\Sigma_{1}\right|^{\frac{1}{2}}}{(2 \pi)^{\frac{k}{2}}\left|\Sigma_{0}\right|^{\frac{1}{2}} \exp \left\{-\frac{1}{2}\left(\mathbf{y}-\mu_{0}\right)^{T} \Sigma_{0}^{-1}\left(\mathbf{y}-\mu_{0}\right)\right\}}
\end{aligned}
$$

Using Neyman-Pearson Lemma, the detection probability can be computed by $[11,13]$ :

$$
P_{d}=\mathbf{Q}\left\{\frac{\Delta}{\sigma} \sqrt{1^{T} \Lambda^{-1}} 1+Q^{-1}\left(P_{f a}\right)\right\}
$$

Where $\Delta=\mu_{1}-\mu_{0}, \mathrm{P}_{\mathrm{fa}}$ represents the false alarm probability and missed-detection probability is shown by $\mathrm{P}_{\mathrm{md}}=1-\mathrm{Pd}$.

From equation (10):

$$
\sqrt{{ }^{T} \Lambda^{-1} 1}=\sqrt{\frac{(1-\xi) k+2 \xi}{1+\xi}}
$$

For very large number of sensing, detection probability can be derived as:

$$
\begin{aligned}
& \lim _{k \rightarrow \infty} \sqrt{\left\{\frac{(1-\xi) k+2 \xi}{1+\xi}\right\}} \\
& \lim _{x \rightarrow 0} \sqrt{\left\{1-k e^{-a_{1} x}-(1-k) e^{-a_{2} x}\right\}\left\{1+\frac{D}{x}\right\}} \\
& \lim _{x \rightarrow 0} \sqrt{\left\{\frac{1-k e^{-a_{1} x}}{x}-\frac{(1-k) e^{-a_{2} x}}{x}\right\}\{x+D\}} \\
& =\sqrt{\frac{D\left(h a_{1}-a_{2}-h a_{2}\right)+2}{2}} \\
& \text { Where } \\
& a_{1}=\frac{1}{d_{A 1}} \text { and } a_{2}=\frac{1}{d_{A 2}} .
\end{aligned}
$$

\section{NUMERICAL RESULTS}

In this section, the detector performance in terms of ROC metric is examined under suburban environments. Furthermore, asymptotic behavior of collaborative spectrum sensing is also examined and compared with Gudmundson's exponential correlation model.

In addition, a cluster-based architecture is also proposed to improve the sensing performance under double correlation model.
It is assumed that a very large number of cognitive sensors, placed equidistantly, are sensing TV transmissions.

The parameters are numerical computations are given as: $\Delta=-5.19, \sigma=2.3, \mathrm{D}=100$, false alarm probability $\mathrm{P}_{\mathrm{fa}}=10^{-3}$, detection probability $\mathrm{P}_{\mathrm{d}}=0.9$, short correlation distance $\mathrm{d}_{\mathrm{Al}}=25$ $\mathrm{m}$, long correlation distance $\mathrm{d}_{\mathrm{A} 2}=200 \mathrm{~m}$, strength between short and long correlation distance $=0.2$, total distance travelled by the cognitive sensor $d$, with 20 number of sensing.

Figure [2] compares the performance of the proposed double correlation model with classic exponential correlation model.

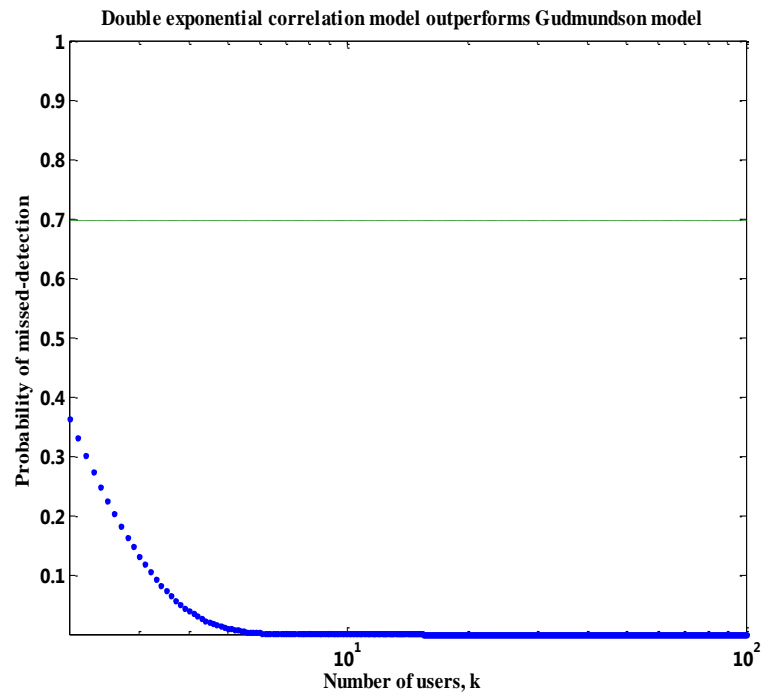

Fig. 2. Verifies the theoretical recommendation that double exponential Correlation model outperforms the Gudmundson's Exponential Correlation Model under suburban environments

\section{Clustered Sensing:}

Clustered sensing corresponds to the case where a number of sensors combine their sensing results for improved detection probability. In this case we consider twenty sensors to submit their results at a fusion center that combines the results of the sensors using OR based combination rule. The following equations can be used to determine detection and false alarm probabilities under suburban environment.

$$
\begin{aligned}
Q_{d} & =\sum_{i=m}^{n}\left(\begin{array}{l}
n \\
i
\end{array}\right)\left(p_{d}\right)^{i}\left(1-p_{d}\right)^{n-i} \\
Q_{f a} & =\sum_{i=m}^{n}\left(\begin{array}{l}
n \\
i
\end{array}\right)\left(p_{f a}\right)^{i}\left(1-p_{f a}\right)^{n-i}
\end{aligned}
$$

Once the probability of detection and false alarm are computed, the results are announced through control channel to all the secondary users so that the cognitive users may benefit from the results appropriately. The results in Figure [3] indicate that even two and three round of sensing results improves detection probability significantly. 


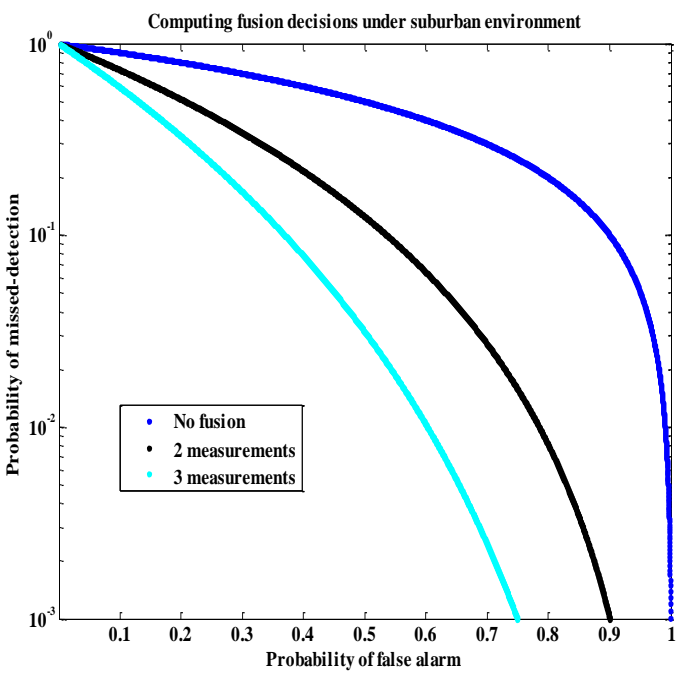

Fig. 3. The improvement in detection performance due to clustered sensing.

\section{CONCLUSION}

Asymptotic performance of collaborative spectrum sensing algorithm is derived and analyzed, using double exponential correlation model under suburban environments. Numerical results have shown that our proposed channel model performs significantly better than classical Gudmundson's exponential correlation model. The proposed model will find wide applications especially in practical wireless systems as the missed-detection metric attains a minimal value very fast (i.e. with minimal number of secondary sensors) in comparison to exponential correlation model. Furthermore, to enhance the performance, a light-weight hard decision combining based strategy for cluster-based detection algorithms is also proposed. This strategy exploits cooperation among sensors to improve detection performance.

Operation of cognitive radio networks mainly in unlicensed RF spectrum poses a threat of interference to the secondary users. Thus, presently, we are studying the performance of spectrum sensors under external interference. The results to this study will indicate the performance metrics under realistic wireless systems.

\section{ACKNOWLEDGMENT}

Authors are thankful to Dr. Muhammad Imran Aslam at NED University of Engineering \& Technology, Karachi for his useful discussions/suggestions.

\section{REFERENCES}

[1] A. Ghasemi and E. S. Sousa, "Asymptotic performance of collaborative spectrum sensing under correlated log-normal shadowing," Communications Letters, IEEE, vol. 11, pp. 34-36, 2007.

[2] A. Tajer and X. Wang, "Beacon-assisted spectrum access with cooperative cognitive transmitter and receiver," Mobile Computing, IEEE Transactions on, vol. 9, pp. 112-126, 2010.

[3] T. Yucek and H. Arslan, "A survey of spectrum sensing algorithms for cognitive radio applications," Communications Surveys \& Tutorials, IEEE, vol. 11, pp. 116-130, 2009.

[4] D. Cabric, et al., "Implementation issues in spectrum sensing for cognitive radios," in Signals, systems and computers, 2004. Conference record of the thirty-eighth Asilomar conference on, 2004, pp. 772-776.

[5] J. G. Proakis, "Digital communications, 1995," ed: McGraw-Hill, New York

[6] Y. Zeng, et al., "A review on spectrum sensing for cognitive radio: challenges and solutions," EURASIP Journal on Advances in Signal Processing, vol. 2010, p. 2, 2010.

[7] E. Visotsky, et al., "On collaborative detection of TV transmissions in support of dynamic spectrum sharing," in New Frontiers in Dynamic Spectrum Access Networks, 2005. DySPAN 2005. 2005 First IEEE International Symposium on, 2005, pp. 338-345.

[8] A. Ghasemi and E. S. Sousa, "Opportunistic spectrum access in fading channels through collaborative sensing," Journal of Communications, vol. 2, pp. 71-82, 2007.

[9] M. Gudmundson, "Correlation model for shadow fading in mobile radio systems," Electronics letters, vol. 27, pp. 2145-2146, 1991.

[10] A. Algans, et al., "Experimental analysis of the joint statistical properties of azimuth spread, delay spread, and shadow fading," Selected Areas in Communications, IEEE Journal on, vol. 20, pp. 523-531, 2002.

[11] A. Ghasemi and E. S. Sousa, "Asymptotic performance of collaborative spectrum sensing under correlated log-normal shadowing," Communications Letters, IEEE, vol. 11, pp. 34-36, 2007.

[12] IEEE 802.22 "Working Group on Wireless Regional Area Networks (WRAN)," IEEE Tech. Rep.

[13] A. Z. Shaikh and T. Altaf, "Performance Analysis of Correlated Multiple Antenna Spectrum Sensing Cognitive Radio," International Journal of Computer Applications, vol. 50, 2012. 